\title{
Forum "Sauvetage en Suisse» de la FMH
}

Le forum «Sauvetage en Suisse» de la FMH, auquel participent tous les représentants des sociétés spécialisées jouant un rôle dans le sauvetage, a récemment établi les critères de qualité du service médical d'urgence, ainsi qu'une description des qualifications des professionnels de la chaîne de sauvetage. Ces critères doivent servir de ligne de conduite, dans le but de promouvoir la qualité de l'organisation future du service médical d'urgence. Ils ont également valeur de déclaration à l'intention de nos partenaires dans le domaine du sauvetage, des autorités et des assureurs.

Concernant les modalités du forum, on a consciemment renoncé à entrer dans les détails, dans le but de permettre aux sociétés cantonales de médecine d'adapter, comme par le passé, le service d'urgence à l'infrastructure, à la topographie et au personnel dans leur domaine de responsabilité. D'autre part, les sociétés de discipline médicale conservent ainsi la souplesse nécessaire à la conception des cours en médecine d'urgence.

Le souhait a souvent été exprimé d'établir une liste standard d'équipement pour le médecin de service et de l'annexer à la liste des critères de qualité. Cette liste doit, elle aussi, être comprise comme une ligne de conduite pouvant être adaptée aux particularités locales. Elle s'inspire des dernières recommandations de l'American Heart Association et sera publiée dans un prochain numéro.

Dr Reto Laetsch, président Forum "Sauvetage en Suisse» de la FMH 